\title{
A Platform-Independent UML Profile for Aspect-Oriented Development
}

\author{
Joerg Evermann \\ Adrian Fiech \\ Farhana Eva Alam \\ Memorial University of Newfoundland Memorial University of Newfoundland Memorial University of Newfoundland \\ St. John's, NL, Canada \\ St. John's, NL, Canada \\ St. John's, NL, Canada \\ jevermann@mun.ca \\ afiech@mun.ca \\ fevalam@mun.ca
}

\begin{abstract}
Aspect Oriented Software Development (AOSD) has its roots in the need to deal with requirements that cut across the primary modularization of a software system. On the programming level, mature, industrial-strength tools like the de-facto standard AspectJ exist. However, on the modeling level, there is as yet little support for AOSD. Building on previous work and using standard UML extension mechanisms, we develop UML modeling support for both static and dynamic AOSD. Code generation is done by working from the UML XMI (XML Model Interchange) format, the standard UML serialization. As a proof-of concept, we implement XSL transformations that generate valid code for two target languages, AspectJ and AspectS.
\end{abstract}

\section{Categories and Subject Descriptors}

D.2.10 [Software Engineering]: Design - Representation

\section{General Terms}

Design, Languages

\section{Keywords}

Aspect-Oriented System Development, Aspect-Oriented Modeling, UML Profile.

\section{INTRODUCTION}

Aspect Oriented Software Development (AOSD) deals with requirements that cut across the primary modularization of a software system, e.g. logging, tracing, security, or persistence. Initially developed as aspect-oriented programming (AOP) [17], it has led to a number of mature tools for different languages. AspectJ [17] for Java, AspectC++ for C++ [24], Aspect\# for C\# [23], AspectS [13] for SmallTalk/Squeak [25] and AspectML [7] for ML are some of the popular AOP language implementations.

The core concepts of AOP are join points, pointcuts, advices and aspects. A join point is a point in the control flow of an application to which additional behavior can be attached, e.g. a call to a method, or the handling of an exception. A pointcut selects join points and collects program execution context at those points. Advices specify behavior to be attached to a join point that

\footnotetext{
Permission to make digital or hard copies of all or part of this work for personal or classroom use is granted without fee provided that copies are not made or distributed for profit or commercial advantage and that copies bear this notice and the full citation on the first page. To copy otherwise, to republish, to post on servers or to redistribute to lists, requires prior specific permission and/or a fee.

$\mathrm{C}^{3} \mathrm{~S}^{2} \mathrm{E}-11$ 2011, May 16-18, Montreal [QC, CANADA]

Editors: Abran, Desai, Mudur

Copyright (C2011 ACM 978-1-4503-0626-3/11/05 \$10.00
}

has been selected by a pointcut. The collection of one or more pointcuts and advices is termed an aspect. In addition to affecting dynamic behavior using advice, it may be possible for aspects to also affect static program structure, e.g. by introducing new class members.

Each AOP implementation has its own Join Point Model (JPM) which defines three things: (i) when the advice can run, (ii) a way to specify pointcuts and (iii) a means of specifying code to run at a join point. JPMs of different AOP implementations can be compared based on the join points exposed, how pointcuts are specified, the operations permitted at join points, and the structural changes that can be expressed.

Aspect-oriented modeling (AOM) supports modularization of cross-cutting concerns at the software design level. Most AOM techniques focus on providing modeling capabilities for the core AOSD concepts, usually as extensions to the Unified Modeling Language (UML). UML provides an extension mechanism known as profile. Models that apply profiles can be processed by any UML tool [22].

Most of the prior work on AOM extends UML either by introducing new meta-model classes or new notation elements without providing meta-level support. Furthermore, many of the existing AOM approaches are programming language specific and thus, in the terminology of Model Driven Architecture (MDA) allow modeling only of platform specific models (PSM), where a platform is understood here as a specific target language for implementation.

The objective of this research is to develop platform independent modeling (PIM) support as a UML profile for the AOP paradigm, and to develop a transformation from a PIM to platform-specific code.

In order to capture a variety of AOSD language features for a PIM profile, we examine two very different AOSD implementations, AspectJ and AspectS. The former is an example of a static AOP implementation using special language constructs, while the latter is an example of a dynamic AOP implementation, using the existing class and object constructs also for AOSD.

The next section introduces our running example in AspectJ and AspectS (Section 2). We then discuss static versus dynamic AOSD (Section 3). This is followed by a comparison of the different features found in AspectJ and AspectS (Section 4), as a preparation for defining a UML profile that allows us to express a core set of AOSD features in UML (Section 5). In Section 6 we illustrate an application of the generic profile. Thereafter we briefly discuss the code generation (Section 7) before concluding with an overview of related work (Section 8) and general discussion (Section 9). 


\section{RUNNING EXAMPLE}

A shopping cart example [18] is implemented using the classes Item, Inventory, ShoppingCart, and ShoppingCartOperator. The ShoppingCartOperator class has two static methods, addShoppingCartItem() and removeShoppingCartItem(). A crosscutting concern for this base system might be the logging of all shopping cart activity. Without aspects, logging code is required for all shopping cart related methods, leading to the problem of code scattering which negatively impacts software reuse and maintenance. We briefly show how aspects avoid this problem.

\subsection{AspectJ Implementation}

AspectJ [17] is a language extension to the Java programming language. It includes the following new constructs: aspect, join point, pointcut, advice, and introduction. When implementing our example in AspectJ, no logging code is added to the base system. Instead, this crosscutting concern is modularized in an aspect named TraceAspect (Listing 1). The pointcut named traceMethods() selects all the method execution join points specified by the wildcards, except those within the lexical scope of TraceAspect itself. The before() advice adds logging code before each method execution specified by the pointcut traceMethods().

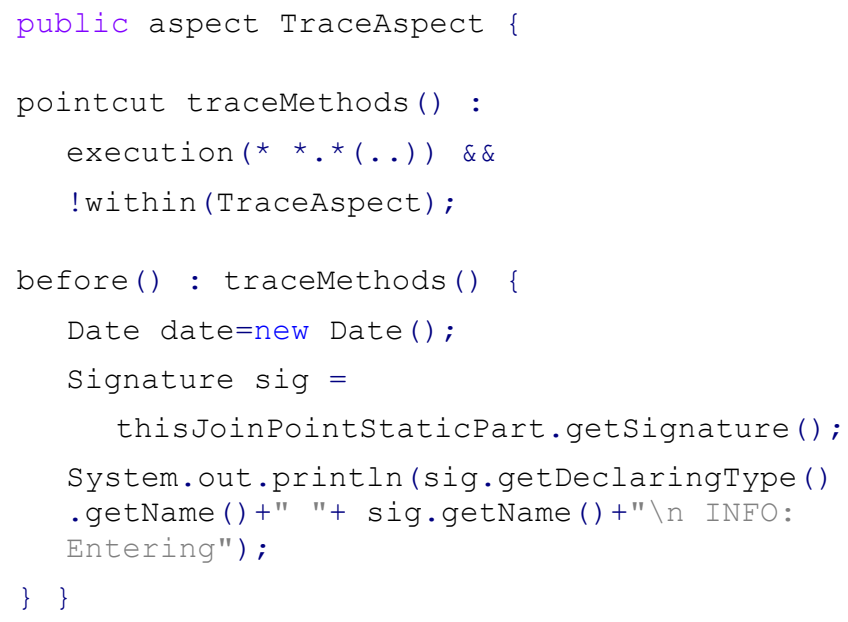

Listing 1 TraceAspect Aspect in AspectJ

\subsection{AspectS Implementation}

AspectS [13] is an AOP implementation for Smalltalk that draws on AspectJ [17] and John Brant's MethodWrappers [6]. The crosscutting-concerns for this example are implemented using an aspect named AspectTrace. The method adviceLogging returns a receiver-class-specific advice that places logging code before and after each join point (Listing 2), and is equivalent to the AspectJ implementation above (details in Section 4). This method is called automatically when installing the aspect at runtime.

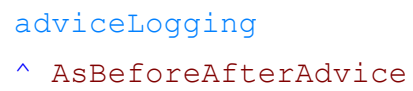

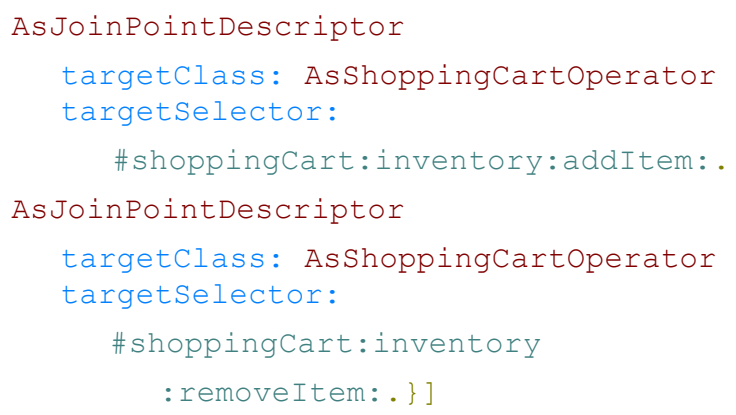

Listing 2 The adviceLogging method in AspectS

\section{AOP APPROACHES: STATIC AND DYNAMIC}

Static AOP, as implemented in AspectJ [17], requires the specification of pointcuts, advice, and aspects at compile time. A weaving compiler adds advice code to join points. When several aspects match the same join point $[4,12]$, they are woven in a predefined order. Aspects cannot be added, removed, or modified at runtime [4]. To change the aspects, the system must be recompiled and restarted, which is not feasible for many systems [10].

Static AOP requires recompilation and restarting of the program when aspects chang or are enabled or disabled. This is not always possible, e.g. when capturing tracing or profiling information on a running production system. Dynamic AOP provides support for controlling aspects at runtime, allowing changes to aspects without restarting the program [4]. A run-time weaver adds advice code to the selected join points. The base system and aspect extensions can be enabled and disabled separately, e.g. from a separate control thread. Dynamic AOP removes AOP overhead when aspects are not required and allows dynamic configuration of aspect behavior, e.g. switching from tracing to profiling, without resetting the state of the base systems. In many implementations of dynamic AOP, the core AOSD concepts are provided using the primary modularization notions, i.e. objects and classes.

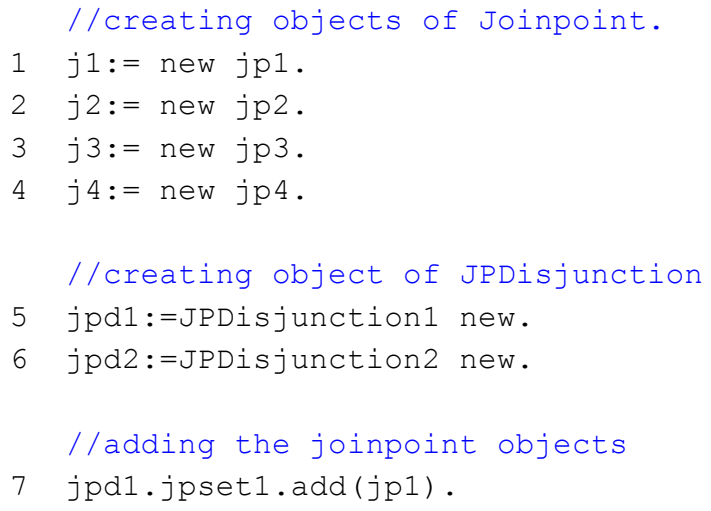




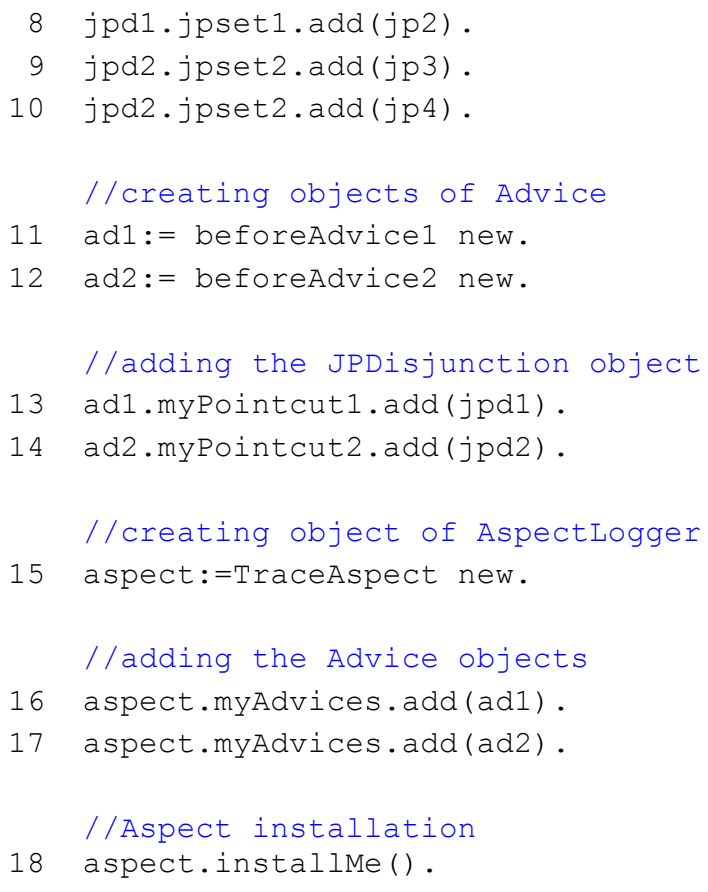

Listing 3 Pseudo code example of dynamic AOP

Listing 3 briefly demonstrates dynamic AOP in pseudocode, beginning with the creation of instances of joinpoint selectors (pointcuts) (lines 1-4), creating two pointcut disjunctions ("or" combinations of pointcuts) (lines 5-6), then inserting the pointcuts into those collections (lines 7-10), creating advice objects (lines 11-12), adding the disjunct pointcuts to the advices (lines 13-14), and finally creating an aspect instance (line 15) and adding the advices to it (lines 16-17), before installing the aspect instance at runtime (line 18). Listing 3 is a motivating example to show the flexibility of dynamic AOP and in our profile we wish to capture the full range of behaviour as illustrated there.

\section{AOP LANGUAGE FEATURE COMPARISON}

Different languages provide different sets of AOSD constructs. To define a platform independent profile for UML, this section identifies commonalities and differences between two representative AOSD implementations.

Table 1 Exposed join point categories in AspectJ and AspectS

\begin{tabular}{|l|l|l|}
\hline & AspectJ & AspectS \\
\hline Method Execution & $\sqrt{ }$ & $\sqrt{ }$ \\
\hline Method Call & $\sqrt{ }$ & $\times$ \\
\hline Constructor Execution & $\sqrt{ }$ & $\times$ \\
\hline Constructor Call & $\sqrt{ }$ & $\times$ \\
\hline Field Read Access & $\sqrt{ }$ & $\times$ \\
\hline Field Write Access & $\sqrt{ }$ & $\times$ \\
\hline Exception handler execution & $\sqrt{ }$ & $\times$ \\
\hline Class initialization & $\sqrt{ }$ & $\times$ \\
\hline Object initialization & $\sqrt{ }$ & $\times$ \\
\hline Object pre-initialization & $\sqrt{ }$ & $\times$ \\
\hline Advice execution & $\sqrt{ }$ & $\times$ \\
\hline
\end{tabular}

An in-depth discussion of the specific differences between the language is beyond the scope of this paper. For reasons of space, we can only briefly highlight the main commonalities and differences that are relevant to our proposed UML profile. These are summarized in three tables.

Table 2 AspectJ joinpoints that are not available in AspectS

\begin{tabular}{|l|l|l|}
\hline preinitialization() & initialization() & within() \\
\hline withincode() & Staticinitialization() & if() \\
\hline
\end{tabular}

Table 1 provides a summary of the comparison of AspectJ and AspectS whereas Table 2 shows AspectJ joinpoints, which are not available in AspectS. Table 3 is a summary of whether AspectS advice qualifier semantics can be expressed in AspectJ.

Table 3 Emulating AspectS advice qualifiers in AspectJ

\begin{tabular}{|c|c|c|}
\hline \multicolumn{2}{|c|}{ Aspect S AdviceQualifier Attributes } & \multirow{2}{*}{$\begin{array}{c}\text { Can be } \\
\text { emulated in } \\
\text { AspectJ }\end{array}$} \\
\hline $\begin{array}{l}\text { Receiver or sender aware } \\
\text { activation }\end{array}$ & cflow activation & \\
\hline receiverClassSpecific & - & $\sqrt{ }$ \\
\hline senderClassSpecific & - & $\sqrt{ }$ \\
\hline receiverInstanceSpecific & - & $x$ \\
\hline senderInstanceSpecific & - & $x$ \\
\hline receiverClassSpecific & Class First & $\sqrt{ }$ \\
\hline receiverClassSpecific & Class All-But-First & $\sqrt{ }$ \\
\hline receiverClassSpecific & Instance First & $x$ \\
\hline receiverClassSpecific & Instance All-But-First & $x$ \\
\hline receiverClassSpecific & Super First & $\sqrt{ }$ \\
\hline receiverClassSpecific & Super All-But-First & $\sqrt{ }$ \\
\hline
\end{tabular}

\subsection{Cross-cutting Concerns}

Each AOP language has the concept of a cross-cutting concern. In AspectJ and AspectS, cross-cutting concerns contain aspects in the same way as packages (AspectJ) or categories (AspectS) contain classes.

\subsection{Aspects}

In AspectS, the user creates instances of aspects. Weaving occurs by sending an install message to an instance of an aspect. In AspectJ, an aspect is a singleton, and an instance is created automatically [18]. For example, when Listing 1 is compiled and executed, an instance of TraceAspect is automatically created. In contract, Listing 2 shows the dynamic creation of an advice object, part of an aspect, by the function adviceLogging. This function creates and returns an instance of AsBeforeAfterAdvice, which the weaver then weaves into the base program.

In AspectJ, the precedence order of advices is explicitly declared at compile-time whereas in AspectS, it is given by the order of install messages sent to aspect instances. In both languages, aspects can be abstract. In AspectJ, aspects can extend classes and abstract aspects $[4,18]$. In AspectS, aspects can inherit from concrete and abstract aspects.

\subsection{Join Points and Pointcuts}

AspectJ exposes method calls and executions as join points whereas AspectS exposes only method executions. Listing 1 
(AspectJ) shows the execution() specification, while this is specified in Listing 2 (AspectS) as part of the pointcut: argument, whose constructor method in turn accepts multiple joinpoint descriptors, each specified by a targetClass and targetSelector field. Unlike AspectJ, AspectS does not expose field access as join points (if fields have accessor methods, those methods can be selected).

AspectJ provides join points for class initialization, object preinitialization and object-initialization (constructor). Since Smalltalk does not know any of these concepts, they cannot be exposed as join points in AspectS.

In contrast to AspectS, AspectJ does not allow instance specific join point selection, e.g. as in the advice qualifier attributes receiverInstanceSpecific and senderInstanceSpecific of AspectS.

AspectJ provides control-flow based join point specifications with cflow() and cFlowBelow(). AspectS uses the advice qualifier attributes cfFirstClass, cfAllButFirstClass, cfFirstInstance, cfAllButFirstInstance, cfFirstSuper and cfAllButFirstSuper to specify control-flow based pointcuts. Some AspectS join points can be emulated in AspectJ by appropriate combinations of control-flow based join points. However, control-flow based instance specific pointcuts of AspectS such as instanceFirst and instanceAllButFirst cannot be emulated in AspectJ.

Lexical-structure based pointcuts, such as within() and withincode() of AspectJ, have no equivalent in AspectS. Listing 1 shows an example of the within() pointcut selector to trace all method executions except those in the aspect itself.

The AspectJ execution object pointcuts this() and target() can be described using advice qualifier attributes in AspectS and vice versa.

The $\operatorname{args()}$ pointcut of AspectJ is used to match join point signatures and is required to expose the context at the matched join point to the advice. AspectS exposes execution context automatically.

In AspectJ, the conditional check pointcut if() captures join points based on some conditions. The conditions are to be checked at the shown in Listing 1, where the within() pointcut selector is negated. Because joint points in AspectS are sets of objects, the generic set methods difference:, union: and intersection: can be applied to AsJoinPointDescriptor objects in AspectS. Listing 2 shows an example, where the pointcut field of the AsBeforeAfterAdvice is the union of two AsJoinPointDescriptor objects.

\subsection{Advice}

AspectJ supports three types of advices: before, after, and around advice. A before advice adds behavior prior to executing a particular join point, as shown in Listing 1. This acts similar to an AsBeforeAfterAdvice with a before code block in AspectS, as shown in Listing 2. The after advice of AspectJ, which adds behavior after executing a join point, can be imitated in AspectS by an AsBeforeAfterAdvice with an after block in it. In AspectJ, before and after advice can be used in the same aspect, and a AsBeforeAfterAdvice may have both the before and after code blocks. An around advice of AspectJ is similar to the AsAroundAdvice of AspectS.

\subsection{Static Crosscutting}

In AspectJ, the introduction construct adds fields to classes and introduces generalization and interface implementation relationships. The AsIntroductionAdvice of AspectS provides similar functions, except that type-hierarchy modification by means of adding generalization relationships is not possible in AspectS.

\section{A PLATFORM INDEPENDENT PROFILE FOR AOSD}

This section presents modeling elements for a platform independent profile for AOSD, based on the common features of AOP languages identified in Section 4.

\subsection{CrossCuttingConcern}

The model element CrossCuttingConcern groups related aspects and is defined as a stereotype that extends the UML meta-class Package.

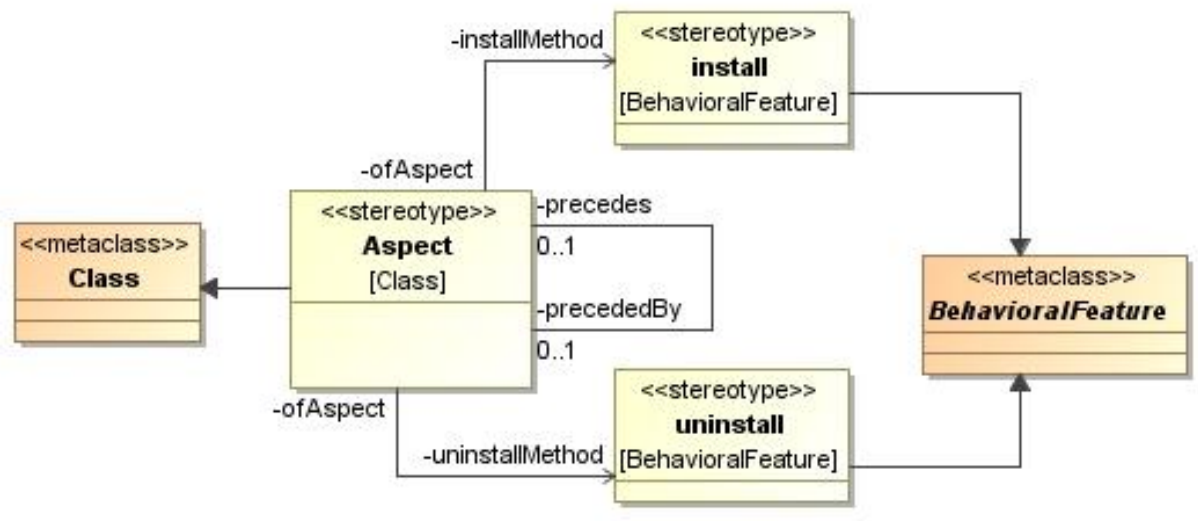

Figure 1 Aspect and dynamic weaving in the generic profile

join point. AspectS does not allow join point selection based on run-time checking.

\subsection{Pointcut operators}

AspectJ provides negation (!), conjunction (\&\&) and disjunction ( $\|)$ to form more complex matching rules [18]. An example is

\subsection{Aspect}

Aspect (Figure 1) is a stereotype that extends the UML meta-class Class. In a model, within a package stereotyped as «CrossCuttingConcern», classes stereotyped as «Aspect» represent aspects. Aspect precedence is modeled as a recursive 
relationship between aspects. Each aspect has at most one directly preceding and following aspect.

\subsection{Install and Uninstall}

To model runtime installation and uninstallation of aspects, we introduce the meta-classes install and uninstall which extend the meta-class BehavioralFeature (Figure 1).

\subsection{Advice}

The meta-class Advice extends Class and is associated with AdviceCollection, which extends StructuralFeature (Figure 2). Modelling advice collections allows assigning multiple advice
We model the selection of execution join points using the metaclass ExecutionJoinpoint. The attribute operation identifies the method whose execution is selected. The attribute senderClass optionally specifies a specific class as message sender or caller. We model exception join points using the meta-class ExceptionJoinpoint. The attribute exceptionClass of type Class is used to specify the class of the exception.

PropertyJoinpoint is the class of join points associated with reading and writing fields. It possesses a multi-valued attribute field of type Property to identify the selected field in the base model.

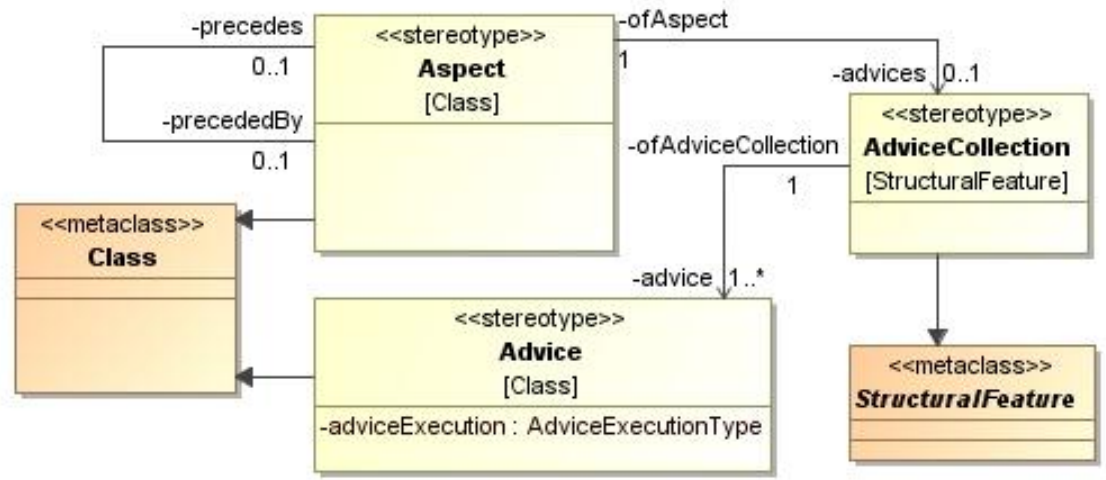

Figure 2 AdviceCollection as a structural feature extension

instances to aspects, as is possible e.g. in ApsectS. adviceExecution is an attribute of Advice, which can take on values 'before', 'after', and 'around'.

\subsection{Pointcut}

To model pointcut instances, the meta-class Pointcut, which extends the StructuralFeature, is introduced. Pointcut is
Based on the discussion of control-flow based join points in Section 4, we introduce a CFlowJoinpoint meta-class. The join points selected by the ClassFirst, ClassAllButFirst, SuperFirst and SuperAllButFirst advice qualifier attributes of AspectS can be selected using a combination of pointcut constructs of AspectJ. Thus, these join points are common to both languages. We model cfPointcut as an attribute of CFlowJoinpoint, with possible values ClassFirst, ClassAllButFirst, SuperFirst, and SuperAllButFirst

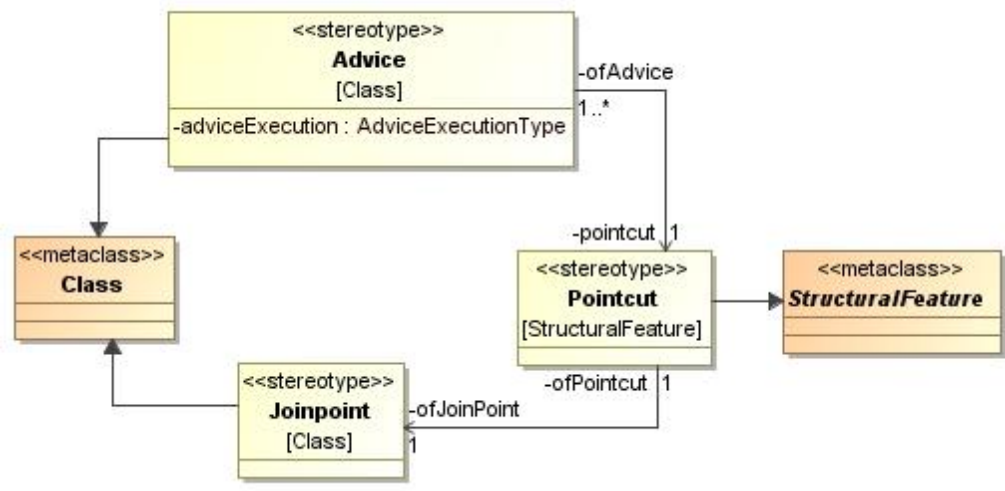

Figure 3 Pointcut as a structural feature extension

associated with Advice (Figure 3). As each pointcut selects a join point, Pointcut is associated with Joinpoint.

\subsection{Join Point}

A join point is modeled using the meta-class Joinpoint, which extends the UML meta-class Class (Figure 3). We specialize the Joinpoint meta-class to model different types of join points. provided by the enumeration CFlowJoinpointType. When the profile is applied, cfPointcut becomes a tag of a stereotyped «AdviceCollection». Joinpoint is associated with CFlowJoinpoint and identifies the join point on which the control-flow based join point is based. 
To provide for join point compositions, we introduce the three meta-classes JoinpointConjunction, JoinpointDisjunction, and JoinpointNegation.

We introduce the meta-class JPCollection to model sets of instances of composed joinpoint specifications. Since join point compositions are based on such sets, JPCollection is associated with both JoinpointConjuction and JoinpointDisjunction. Thus, multiple instances of a composed join point can be modeled, e.g. profile allows for either static or dynamic AOSD, for reasons of space, we illustrate only dynamic AOSD, shown in Figure 6.

The aspect TraceAspect consists of two composed pointcuts, JPDisjunction1 and JPDisjunction2. JPDisjunction1 is composed of execution pointcuts $j p 1, j p 2, j p 3$, and $j p 4$. JPDisjunction 2 is composed of execution pointcuts jp5 and jp6. Pointcuts JPDisjunction1 and JPDisjunction 2 are advised by two before advices. We select the method addItem of the Inventory class

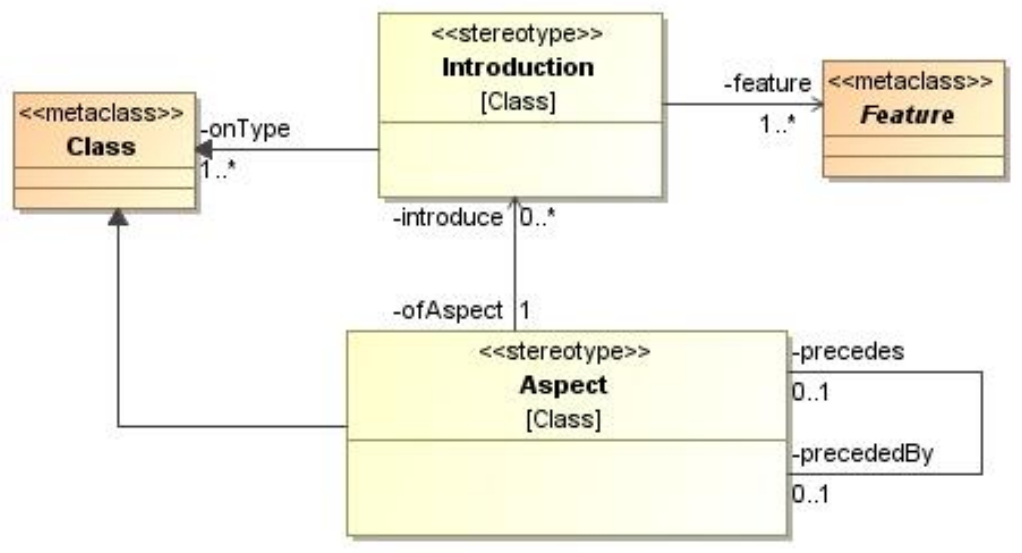

Figure 4 Introduction as a class extension

as shown in the pseudocode of Lines \#7 and \#8 in Listing 3.

Statically introduced features are modeled using the meta-class Introduction, which extends Class. Since such features are introduced by aspects, Introduction is associated with Aspect (Figure 4). To specify the features that are introduced, Introduction possesses the attribute feature, whose type is the UML meta-class Feature. The onType attribute of meta-class Class, specifies the types on which the cross-cutting feature will be introduced.

Figure 5 presents the final PIM profile for AOP languages. The profile allows the modeler to model either static or dynamic AOP, but not in the same model. It represents the union of common features in AspectS and AspectJ and those that may not have a simple equivalent, but can be generated with equivalent semantics (e.g. while the introduction of AspectJ is a basic feature, it can be specified using appropriate aspects in AspectS). The user of the profile need not specify the target platform until time of code generation.

However, the modeler does need to be specific about whether static or dynamic AOP is targeted. For example, for static AOP, install and uninstall methods must not be specified, whereas dynamic AOP requires the JPCollection element to collect joinpoints created dynamically at runtime.

The appendix specifies additional constraints in the Object Constraint Language (OCL) that form part of the profile and ensure that profile applications are internally consistent. Among other things, the constraints help to prevent the mixing of static and dynamic AOP elements in the same profile application.

\section{APPLICATION EXAMPLE}

In Section 2 we introduced the shopping cart example. We use it here to show the application of the generic profile. While our from the base model as the value of operation of $j p 1$, indicating that the execution of this method is selected as a join point. Similarly, the methods removeItem of class Inventory, addItem of ShoppingCart, removeItem of ShoppingCart, addShoppingCartItem of ShoppingCartOperator, and removeShoppingCartItem of ShoppingCartOperator are selected as the values of operation of jp2,jp3,jp4, jp5 and jp6, respectively. The hasParts attributes of the stereotyped «JoinpointDisjunction» classes specify the join points that are parts of the compositions.

As shown in Figure 6, we model all the elements, such as crosscutting concern (package), aspect, and join points. We introduce a class CollectionType, e.g. a Java collection type, which collects advice or aspect instances. Hence, it is associated with the aspect and advice classes. The values of ofJoinpoint 1 of myPointcut 1 and ofJoinpoint2 of myPointcut2 are respectively set to JPDisjunction1 and JPDisjunction2. Also, the value of pointcut of beforeAdvicel and beforeAdvice2 are respectively set to myPointcut 1 and myPointcut 2 so that the advice instances "know" about the collections they are collected in. TraceAspect is associated with the class CollectionType by its attribute advice, which is stereotyped «AdviceCollection» to indicate that this field will contain a collection of advices. The meta-attribute advice becomes a tag. beforeAdvicel and beforeAdvice 2 are selected as values of the tag as these are the types of advices we collect in the advice collection.

We create two operations installMe and uninstallMe for TraceAspect. When the profile is applied, these operations become stereotyped «install» and «uninstall», and are selected as values of installMethod and uninstallMethod of TraceAspect.

The elements in the presented model can be used as shown in the pseudo code in Listing 3. 


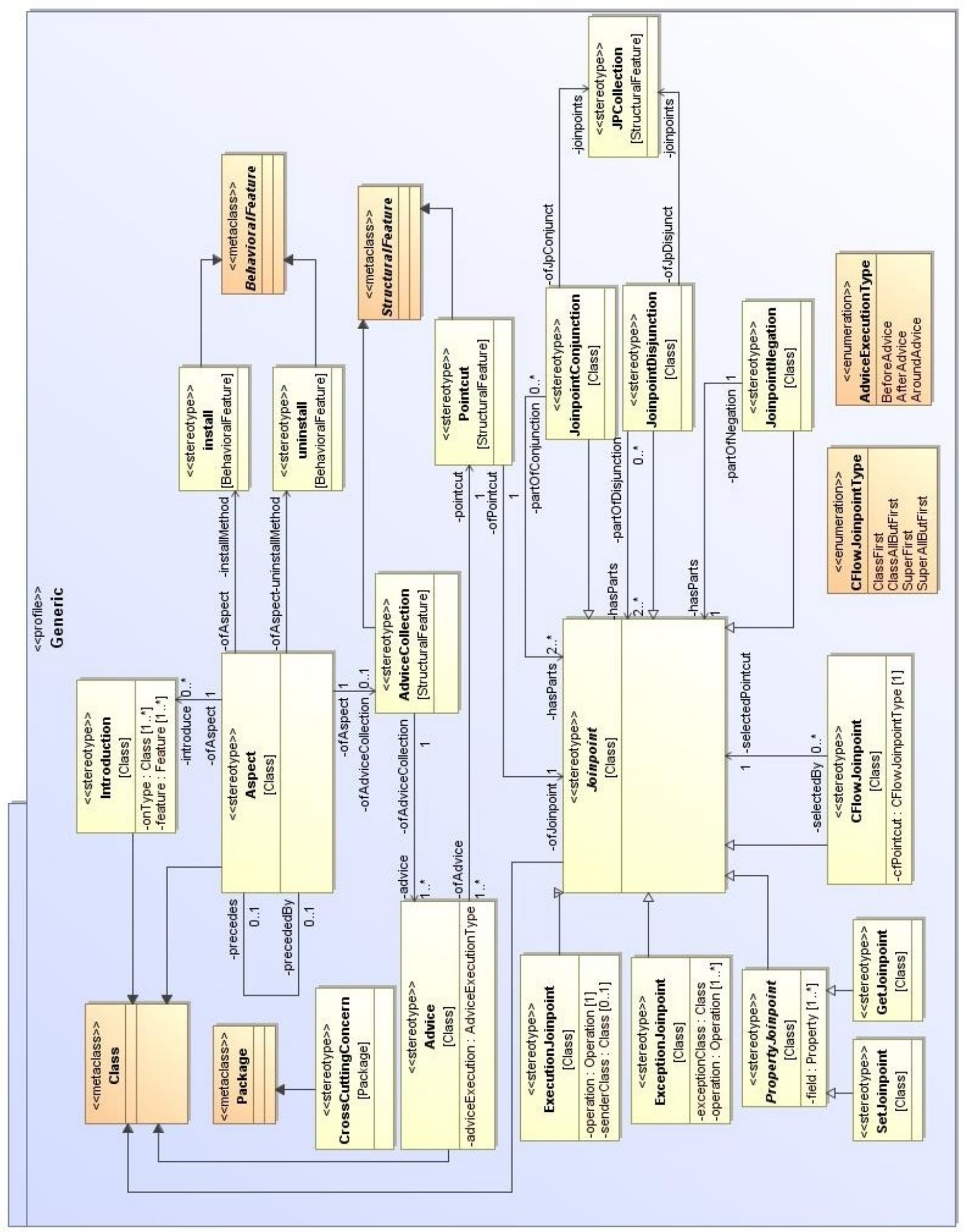

Figure 5 Core generic meta-model for AOP languages 


\section{CODE GENERATION}

Because the profile is compliant with standard UML XMI format and is fully specified in terms of the meta-model, the model to which the profile is applied also becomes compliant with standard advices and pointcuts often remains in textual form $[5,15,21]$, requiring special model parsers for code generation. In contrast, our work is formally based on the meta-model extension mechanism provided by stereotypes and profiles.

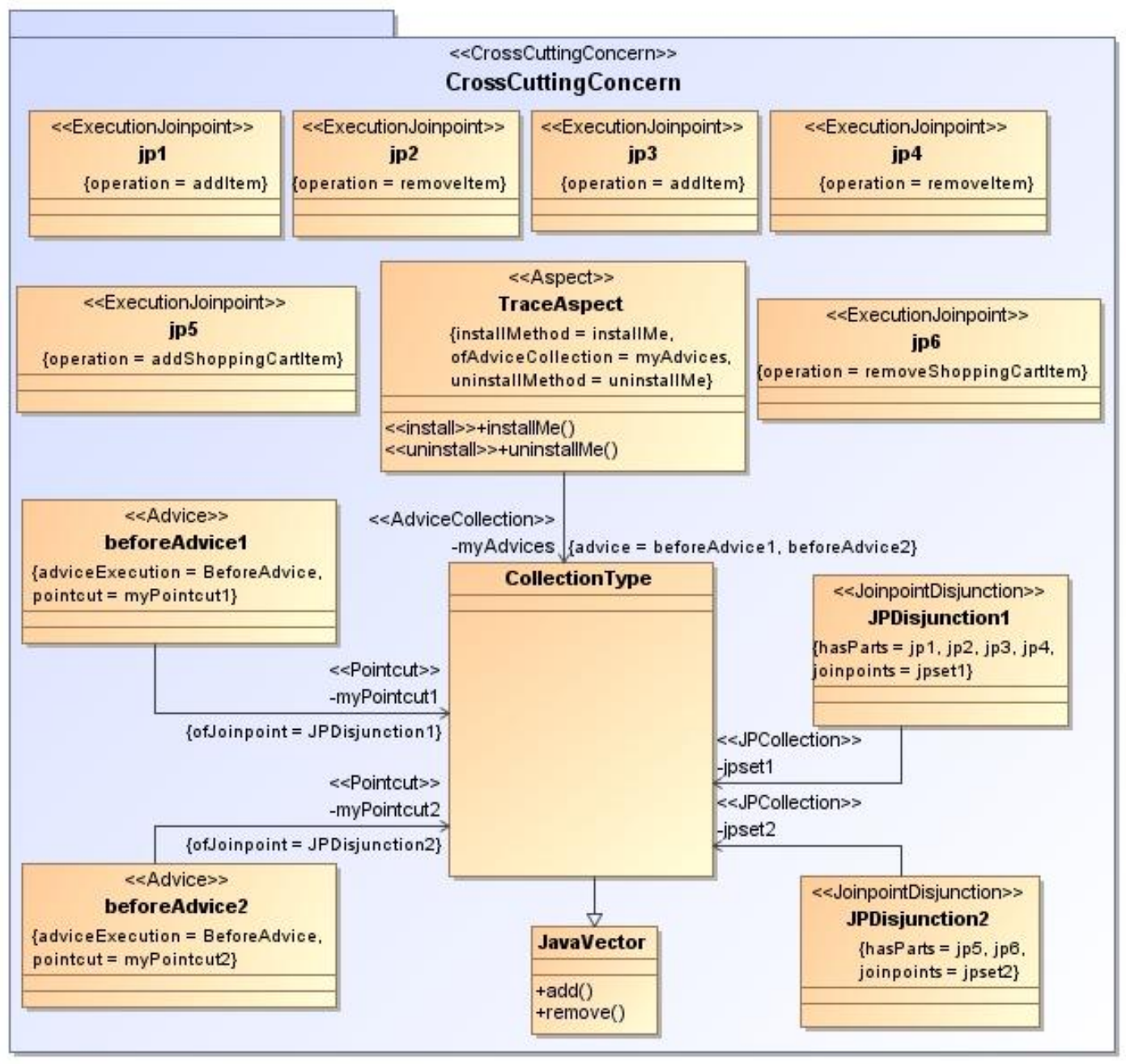

Figure 6 Dynamic cross-cutting concern of the shopping-cart example

UML XMI format. As a result, code can easily be generated. As a proof-of-concept, we have implemented two XSL (XML Stylesheet Language) Transformations (XSLT) that generate valid AspectJ and AspectS code. Code generation for AspectJ is implemented in approximately 1100 lines of XSLT code and consists of 26 templates. Code generation for AspectS is implemented in approximately 1250 lines of XSLT code and consists of 31 templates.

This implementation allowed us to verify our profile as the generated code can be directly compiled (with empty advice stubs). The UML profile as well as the XSLT code is available from the authors upon request.

\section{RELATED WORK}

An overview of some of the prior work for modeling aspects in UML is presented in [1] and [9]. The early work [1] is based on the extension mechanisms in UML 1.x versions. Since these mechanisms are not meta-model based, the specification of
Initial work presented in [2] proposed the specification of aspects as stereotypes on classes and was later extended to include advice and pointcut specification [3]. Because cross-cutting associations show which aspect features relate to which base model elements, there is no clear separation between base system model and crosscutting concerns; both are necessarily part of the same model. Our work improves on this by separating base system model from cross-cutting concerns so that different cross-cutting concerns may be added without necessitating any adaptation of the base model.

A profile for AspectJ is proposed in [26]. It represents messages in collaborations as join points, advices and pointcuts as stereotyped operations, and introduction of fields or methods as templated collaborations. Connections to the base features are made via dependencies in the model [8]. As in [2, 3], this also violates the clear separation of base-system model and crosscutting concerns that underlies the idea of aspect-oriented 
development. Again, our proposed approach addresses this weakness.

Using standard UML extension mechanisms, [27] provides representations for all components of an aspect (such as join points, pointcuts, pieces of advice, and introductions) as well as for the aspect itself. These are supplied with supplementary metaattributes to hold weaving instructions. The approach implements the AspectJ weaving mechanism on the UML model and consequently requires special modeling tool support. In contrast, our approach relies on existing programming-language level weavers without having to re-develop weaving at the model level.

Using the extension mechanisms in UML 2, [8] presents a metamodel that is a profile for AspectJ and offers code translation from XMI. In contrast to our work, that profile is a platformspecific profile that allows only an AspectJ implementation.

A UML 2 profile for platform-independent modeling is presented in [20]. It has advanced pointcut expressions and a corresponding model weaving mechanism for behavior models using UML actions, again requiring special modeling tool support.

Recent work on Aspect-Oriented Frameworks (AOF)-based development is presented in [14]. It proposes UML-AOF, a UML profile for modeling a kind of AOF (termed as CF in the proposal), which encapsulates just one crosscutting concern. However, like [8], the presented profile does not support the specification of platform-independent model.

In summary, much of the existing work on AOM profiles for UML is either based on older UML versions, not well integrated on the meta-model level, is platform specific (typically targeting the AspectJ join point model), or requires special modeling tools for support. In contrast, our proposal targets the current UML specification version, makes full use of the meta-model level extension mechanism (profiles and stereotypes), is platform independent and, instead of requiring special model-level weavers, relies on robust and proven programming-level language weavers. By separating the base-system model and the crosscutting models, our approach allows the two to be developed independently.

\section{DISCUSSION}

Building on previous work [8], this research presents a metamodel, which is also a UML profile, based on the core generic features of AOSD approaches. To cover a variety of AOP features, we examined AspectJ and AspectS, which support static and dynamic AOP. For the UML profile, we have chosen common language features. This profile allows existing UML tools to express AOSD models. Since our profile enables the support for modeling of static or dynamic AOP, it is quite complex. Using separate profiles for static and dynamic AOSD could reduce this complexity.

Applying the profile to models is also complex, as the aspectoriented features must be specified explicitly and explicitly linked to the base system. As a result, the modeller must be aware of the complete base-system model. This can be solved by using pattern based, textual specification as earlier work had done. However, the power of pattern specification is not available in the UML meta-model. Also, while textual, pattern-based specification is convenient, it opens the door to inadvertent selection of unintended join points. This is known as the fragile pointcut problem $[11,16]$ and is especially problematic when refactoring [19] the base system code, since pattern-based pointcut specifications depend strongly on the specific design of the base system [8]. Thus, the explicit specification required by our profile is safer.

From a practitioner's perspective, the lightweight, meta-model based extension mechanisms of UML 2 makes this profile useful, as it is supported by existing, mature UML 2 compliant modelling tools and requires no special software support. The proposed technique is supported by UML XMI model interchange facilities. The model extension, as well as any models it is applied to, can be exchanged between different MOF (Meta-Object-Facility) compliant UML modeling tools. Moreover, because the profile is platform independent, and the weaving relies on programminglanguage level weavers, it is easy to add additional platform (implementation language) support. This can be done simply by adapting the existing XSLT elements to produce e.g. AspectC\# instead of AspectS.

\section{ACKNOWLEDGMENTS}

This research was partially supported by funding from the Natural Sciences and Engineering Research Council of Canada (NSERC) - grant OGP0170497

\section{REFERENCES}

1. Reina A., Torres J., and Toro M.: 'Towards Developing Generic Solutions with Aspects'. Proceedings of the AOM workshop at AOSD, 2004.

2. Aldawud, O., Elrad, T. and Bader, B.: 'A UML Profile for Aspect Oriented Modeling'. Proceedings of OOPSLA 2001.

3. Aldawud, O., Elrad, T. and Bader, A.: 'UML Profile for Aspect-Oriented Software Development' The Third International Workshop on Aspect Oriented Modeling, 2003.

4. Assaf, A. and Noyé, J.: 'Dynamic AspectJ'. Proceedings of the 2008 symposium on Dynamic languages, 1-12, 2008.

5. Basch, M. and Sanchez, A.: 'Incorporating Aspects into the UML', 2003.

6. Brant, J., Foote, B., Johnson, R.E. and Roberts, D.: 'Wrappers to the Rescue'. Proceedings ECOOP '98, 396417, 1998

7. Dantas, D.S., Walker, D., Washburn, G. and Weirich, S.: 'AspectML: A Polymorphic Aspect-Oriented Functional Programming Language'. ACM Trans. Program. Lang. Syst., 30, 1-60, 2008

8. Evermann, J.: 'A Meta-Level Specification and Profile for AspectJ in UML'. Proceedings of the 10th International Workshop on Aspect-Oriented Modeling, 21-27, 2007

9. Fuentes, L. and Sanchez, P.: 'Elaborating UML 2.0 Profiles for AO Design' Proceedings of the AOM workshop at AOSD, 2006.

10. Gilani, W., Scheler, F., Lohmann, D., Spinczyk, O. and Schröder-Preikschat, W.: ,Unification of Static and Dynamic AOP for Evolution in Embedded Software Systems' Proceedings of the Sixth International Symposium on Software Composition, 216-234, 2007

11. Gybels, K. and Brichau, J.: 'Arranging Language Features for More Robust Pattern-based Crosscuts' Proceedings of the 2nd international conference on Aspect-oriented software development, 60-69, 2003

12. Hilsdale, E. and Hugunin, J.: 'Advice Weaving in AspectJ'. Proceedings of the 3rd international conference on Aspectoriented software development, 26-35, 2004

13. Hirschfeld, R.: 'AspectS - Aspect-Oriented Programming with Squeak'. Proceedings of the International Conference NetObjectDays on Objects, Components, Architectures, 
Services, and Applications for a Networked World, 216232, 2003

14. Uetanabara, J., Camargo, V.V. and Von Flach Chavez, C.: 'UML-AOF: A Profile for Modeling Aspect-Oriented Frameworks'. Proceedings of the 13th workshop on Aspectoriented modeling, 1-6, 2009

15. Mancona Kande, M., Kienzle, J. and Strohmeier, A.: From AOP to UML- A Bottom-Up Approach.

16. Kellens, A., Mens, K., Brichau, J., and Gybels, K.: 'Managing the evolution of aspect-oriented software with model-based pointcuts'. Proceedings of the European Conference on Object-Oriented Programming (ECOOP), 501-525, 2006.

17. Kiczales, G., Hilsdale, E., Hugunin, J., Kersten, M., Palm, J. and Griswold, W.G.: 'An Overview of AspectJ' Proceedings of the 15h European Conference on ObjectOriented Programming, 327-354, 2001

18. Laddad, R.: AspectJ in Action: Practical Aspect-Oriented Programming. Manning Publications, 2003.

19. Mens, T. and Tourwé, T.: 'A Survey of Software Refactoring'. IEEE Trans. Softw. Eng., 30, 126-139, 2004

20. Mosconi, M., Charfi, A., Svacina, J. and Wloka, J.: 'Applying and Evaluating AOM for Platform Independent Behavioral UML Models'. Proceedings of the 2008 AOSD workshop on Aspect-oriented modeling, 19-24, 2008

21. Pawlak, R., Duchien, L., Florin, G., Legond-Aubry, F., Seinturier, L., and Martelli, L.: 'A UML Notation for Aspect-Oriented Software Design'. Workshop on AspectOriented Modeling with UML, 2002.

22. Pilone, D. and Pitman, N.: UML 2.0 in a Nutshell. O'Reilly, 2005.

23. Devi Prasad, M. and Chaudhary, B.D.: 'AOP Support for C\#'. AOSD Workshop on Aspects, Components and Patterns for Infrastructure Software, 49-53, 2003

24. Spinczyk, O., Gal, A. and Schröder-Preikschat, W.: 'AspectC++ : An Aspect-Oriented Extension to the C++ Programming Language'. Proceedings of the Fortieth International Conference on Tools, Darlinghurst, Australia, 53-60, 2002.

25. The squeak homepage. http://www.squeak.org/.

26. Stein, D., Hanenberg, S. and Unland, R.: 'Designing Aspect-Oriented Crosscutting in UML'. Proceedings of the AOSD-UML Workshop at AOSD '02, 2002.

27. Stein, D., Hanenberg, S., and Unland, R.: 'An UML-based Aspect-Oriented Design Notation for AspectJ'. Proceedings of the 1st international conference on Aspect-oriented software development, 106-112, 2002

\section{Appendix: OCL Constraints}

This appendix defines a set of constraints in the Object Constraint Language (OCL) that are included in the profile. The OCL is a part of the UML specification and these constraints express invariants that cannot be expressed in the graphical UML models.
For reasons of space, we cannot provide an in-depth discussion of each constraint.

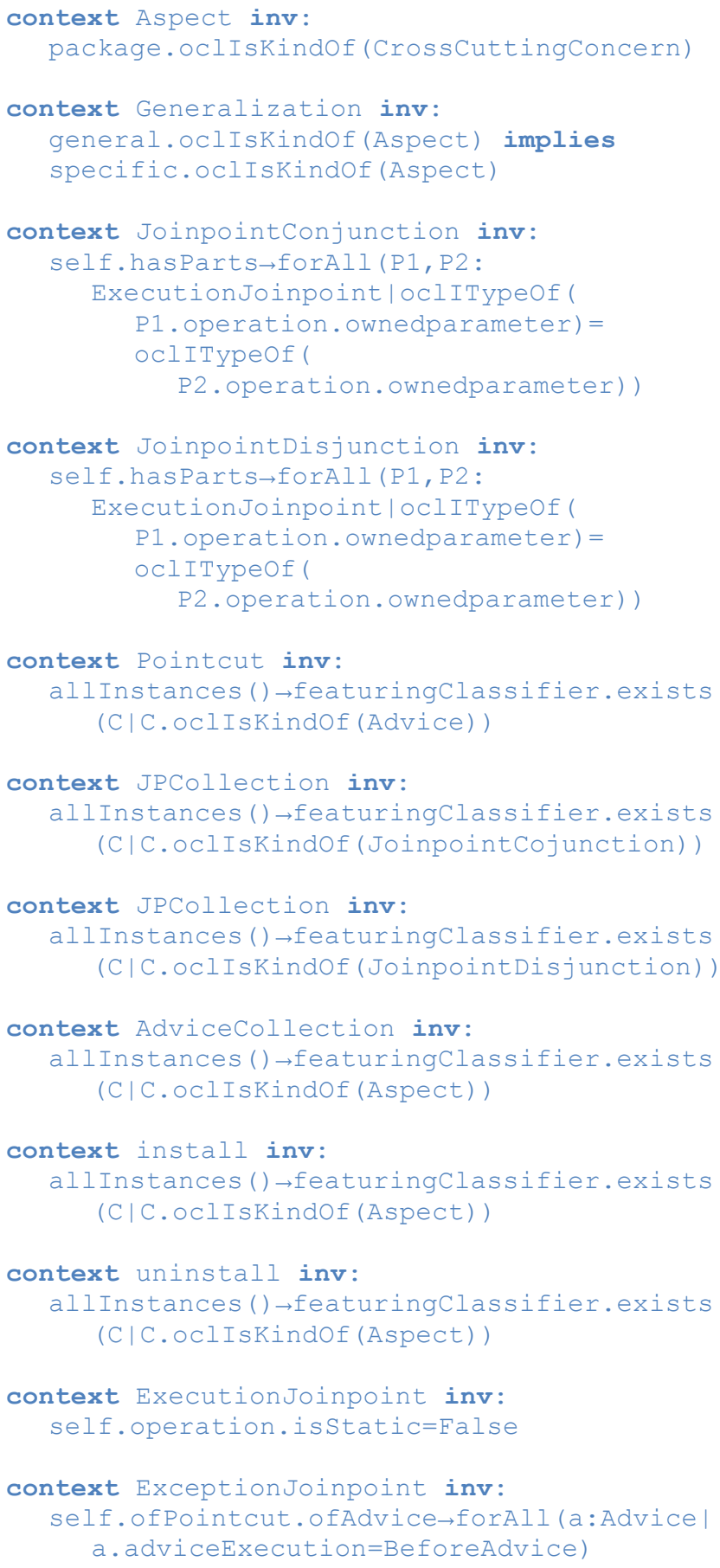

